\title{
APPROXIMATION IN THE MEAN BY ANALYTIC FUNCTIONS
}

\author{
LARS INGE HEDBERG
}

\begin{abstract}
Let $E$ be a compact set in the plane, let $L^{p}(E)$ have its usual meaning, and let $L_{a}^{p}(E)$ be the subspace of functions analytic in the interior of $E$. The problem studied in this paper is whether or not rational functions with poles off $E$ are dense in $L_{a}^{p}(E)$ (or in $L^{p}(E)$ in the case when $E$ has no interior). For $1 \leqq p \leqq 2$ the problem has been settled by Bers and Havin. By a method which applies for $1 \leqq p<\infty$ we give new results for $p>2$ which improve earlier results by Sinanjan. The results are given in terms of capacities.
\end{abstract}

1. Introduction. In recent years the problem of uniform approximation by rational functions in the complex plane has received great attention (see e.g., [26], [28], [11]), but comparatively little work has been devoted to the corresponding problem of approximation in the areal mean. Carleman proved in [5] that if $D$ is a Jordan region, then every function in $L^{2}(D)$ which is analytic in $D$ can be approximated in $L^{2}(D)$ by polynomials, and this result was later extended by Farrell and Markuševič to Carathéodory regions (see [21]). Only much later was the corresponding problem for approximation by rational functions studied. See Bers [2], Sinanjan [22], [23], Havin [15]. In particular, Havin discovered that necessary and sufficient conditions for approximation in $L^{2}$ can be given in terms of logarithmic capacity. He remarked, however, that his method of proof does not easily extend to $L^{p}, p>2$.

In this paper we employ a method of proof which applies to all $p, 1 \leqq p<\infty$, and get new approximation theorems for $p>2$ which improve those of Sinanjan [23]. We also get new proofs of some of the results of Havin [15]. The method of proof goes back to Bers [2], and is similar to that used by the author in [18].

The main results are stated in $\S 3$ of the paper. They are given in terms of analytic $p$-capacity, or equivalently, $q$-capacity, which are defined in $\$ 2$ below, where we also study the relations of these capacities to potential theoretic capacity and "Hausdorff content".

For simplicity we will only consider approximation on bounded sets.

The author is indebted to John Garnett, John Wermer, and the referee for valuable comments.

Presented to the Society April 18, 1970; received by the editors June 3, 1970 and, in revised form, December 30, 1970.

AMS 1969 subject classifications. Primary 3070,3085,4630; Secondary 3041,3121,3122, 3052.

Key words and phrases. Approximation in areal mean, rational functions, $L^{p}$-spaces, compact sets, analytic $p$-capacity.

Copyright (C) 1972, American Mathematical Society 
The following notation will be used. If $E$ is a measurable set, $\bar{E}$ will be its closure, $E^{\circ}$ its interior, $\partial E$ its topological boundary, and $\mathscr{C} E$ its complement. $L_{a}^{p}(E)$ is the closed subspace of $L^{p}(E)$ which consists of functions analytic in $E^{\circ} . R^{p}(E)$ is the closure in $L^{p}(E)$ of rational functions with poles in $\mathscr{C} E$.

For $f \in L^{p}(E),\|f\|_{p}=\left\{\int_{E}|f(z)|^{p} d A\right\}^{1 / p}$, where $d A$ is Lebesgue measure.

$q$ will always mean $p /(p-1)$. The disk $\{\zeta ;|\zeta-z|<\delta\}$ is denoted by $K_{z}(\delta)$. Various constants will be denoted by $K$.

2. Capacities. Let $E$ be a compact set, and let $1<q \leqq 2$. By $\Omega$ we mean the complex plane when $q<2$, and a fixed bounded region containing $E$ in its interior in the case $q=2$. The $q$-capacity $\Gamma_{q}(E)$ is defined in the following way.

Definition. $\Gamma_{q}(E)=\inf _{\varphi} \int_{\Omega}|\operatorname{grad} \varphi|^{q} d A$, where the infimum is taken over all real-valued Lipschitz continuous functions $\varphi$ with support in $\Omega$ such that $\varphi(z) \geqq 1$ on $E$.

If $E$ is arbitrary we define $\Gamma_{q}(E)=\sup _{F} \Gamma_{q}(F), F \subset E, F$ compact.

REMARK. This set function has been studied e.g. by Wallin [27] and Ziemer [29]. Ziemer proved, among other things, that $\Gamma_{q}$ satisfies the conditions in Choquet's theorem, and therefore e.g. all Borel sets are capacitable.

We can also define an analytic $p$-capacity $\gamma_{p}(E), 2 \leqq p<\infty$, in the following way.

Definition. $\gamma_{p}(E)=\sup \left|(1 / 2 \pi i) \int_{c} f(z) d z\right|$, where the supremum is taken over all functions $f$ in $L_{a}^{p}(\Omega \backslash E)$ with $\|f\|_{p} \leqq 1$, and $c$ is a Jordan curve enclosing $E$.

If $E$ is arbitrary we define $\gamma_{p}(E)=\sup _{F} \gamma_{p}(F), F \subset E, F$ compact.

REMARK. A somewhat different definition of analytic $p$-capacity was given by Sinanjan in [23]. This restricts his results unnecessarily, as will be seen below.

Lemma 1. Let $E$ be compact. Then

$$
\gamma_{2}(E)=\frac{1}{2 \pi} \Gamma_{2}(E)^{1 / 2}
$$

For $1<q<2$ there are positive constants $K_{1}$ and $K_{2}$ (only depending on $q$ ) such that

$$
K_{1} \Gamma_{q}(E)^{1 / q} \leqq \gamma_{p}(E) \leqq K_{2} \Gamma_{q}(E)^{1 / q} .
$$

Proof. First assume that $E$ (and $\Omega$ ) has smooth boundary.

Let $p=2$, and let $\omega(z)$ be the harmonic measure of $E$ with respect to $\Omega$. Then, for any $f \in L_{a}^{2}(\Omega \mid E)$ with continuous boundary values (I owe this idea to John Wermer),

Thus

$$
\begin{aligned}
\left|\int_{\partial E} f(z) d z\right| & =\left|\int_{\partial E} f(z) \omega(z) d z\right| \\
& =\left|\int_{\Omega \backslash E} \frac{\partial}{\partial \bar{z}}(f \omega) d \bar{z} \wedge d z\right|=\left|2 i \int_{\Omega \backslash E} f\left(\frac{\partial \omega}{\partial z}\right) d A\right| .
\end{aligned}
$$

$$
\left|\int_{\partial E} f(z) d z\right|^{2} \leqq 4 \int_{\Omega \mid E}|f|^{2} d A \int_{\Omega \mid E}\left|\frac{\partial \omega}{\partial z}\right|^{2} d A
$$


Equality holds for $f=\partial \omega / \partial z$, which is a single-valued analytic function. It follows that

$$
\left|\frac{1}{2 \pi} \int_{\partial E} \frac{\partial \omega}{\partial z} d z\right|=\frac{1}{4 \pi}\|\operatorname{grad} \omega\|_{2}^{2}
$$

and since functions with continuous boundary values are dense it follows that $\gamma_{2}(E)=(1 / 2 \pi) \Gamma_{2}(E)^{1 / 2}$.

Now let $p>2$, and let $\omega(z)$ be any complex-valued Lipschitz function with compact support such that $\omega(z)=1$ on $E$. We obtain in the same way

$$
\left|\int_{\partial E} f(z) d z\right|=\left|2 i \int_{\mathscr{C} E} f \frac{\partial \omega}{\partial \bar{z}} d A\right| \leqq 2\|f\|_{p}\left\|\frac{\partial \omega}{\partial \bar{z}}\right\|_{q} .
$$

It follows that $\gamma_{p}(E) \leqq K \Gamma_{q}(E)^{1 / q}$. The general case follows easily.

To get the other inequality we will use a standard Hahn-Banach argument (see e.g. Havinson [17]). We note that by the above $\alpha(f)=(1 / 2 \pi i) \int_{\partial E} f(z) d z$ is a bounded linear functional on $L_{a}^{p}(\mathscr{C} E)$ represented by

$$
\frac{1}{\pi}\left(\frac{\partial \omega}{\partial \bar{z}}\right)=\frac{1}{\pi} \frac{\partial \bar{\omega}}{\partial z}
$$

On the other hand, it is known (see e.g. [16, Lemma 1]) that functions of the form $\partial \varphi / \partial z$, with $\varphi \in C_{0}^{\infty}(\mathscr{C} E)$ form a dense subset of the annihilating space of $L_{a}^{p}(\mathscr{C} E)$. Thus, by the Hahn-Banach theorem, there are Lipschitz functions $\omega$ with compact support, with $\omega=1$ on $E$, such that $(1 / \pi)\|\partial \omega / \partial z\|_{q}$ is arbitrarily close to the norm of the functional $\alpha$, i.e. to $\gamma_{p}(E)$. It follows that $\gamma_{p}(E)=(1 / \pi)$ inf $\|\partial \omega / \partial z\|_{q}$, for such functions $\omega$. The lemma follows since $\|\operatorname{grad}|\omega|\|_{q} \leqq K\|\partial \omega / \partial z\|_{q}$ for functions with compact support and $q>1$, by the Calderón-Zygmund theory [4].

We will also examine the relationship between $\gamma_{p}(E)$ (and $\Gamma_{q}(E)$ ) and potential theoretic capacity $C_{q}(E)$ and "Hausdorff content" $M_{q}(E)$. If $\mu$ is a measure on $E$ we denote the potential of $\mu$ with respect to Green's function for $\Omega$ by $U_{2}^{\mu}$, and the potential with respect to a kernel $r^{q-2}, 1<q<2$, is denoted by $U_{q}^{\mu}$. Then for $1<q \leqq 2, C_{q}(E)=\sup \mu(E)$, the supremum being taken over positive measures on $E$ with $\sup _{z} U_{q}^{\mu}(z) \leqq 1$.

For an increasing function $h(r), h(0)=0$, we define the "Hausdorff content" $M_{h}(E)=\inf \sum_{i} h\left(r_{i}\right)$, the infimum being taken over all coverings of $E$ by disks with radii $r_{i}$. For $h(r)=r^{2-q}, 1<q<2$, we write $M_{q}(E)$. For the properties of $M_{h}$ and its relation to capacities we refer to [7].

The following lemma is essentially contained in [7, Theorem VI:1].

Lemma 2. Let E be compact with connected complement. Then

$$
\gamma_{2}(E)=(2 \pi)^{-1 / 2} C_{2}(E)^{1 / 2} .
$$

For $1<q<2$ there are positive constants $K_{1}$ and $K_{2}$ (only depending on $q$ ), such that

$$
K_{1} C_{q}(E)^{1 / q} \leqq \gamma_{p}(E) \leqq K_{2} M_{q}(E)^{1 / q} .
$$


Proof. First let $p=2$, and let $\omega$ be the harmonic measure for $E$ with respect to $\Omega$. Then $\omega$ can be represented as a potential, $\omega(z)=\int_{E} G(z, \zeta) d \mu(\zeta)$, where $G$ is Green's function for $\Omega$, and $\mu$ is the corresponding equilibrium measure for $E$ with total mass $C_{2}(E)$. It follows that, for any Jordan curve $c$ enclosing $E$,

$$
\begin{aligned}
& \int_{c} \frac{\partial \omega}{\partial z} d z=\int_{c}\left\{\int_{E} \frac{\partial G(z, \zeta)}{\partial z} d \mu(\zeta)\right\} d z=\int_{E}\left\{\int_{c} \frac{\partial G}{\partial z} d z\right\} d \mu(\zeta) \\
& =\int_{E}\left\{\int_{c} \frac{\partial}{\partial z} \log \frac{1}{|z-\zeta|} d z\right\} d \mu(\zeta)=\frac{1}{2} \int_{E}\left\{\int_{c} \frac{d z}{\zeta-z}\right\} d \mu(\zeta)=-\pi i \mu(E)=-\pi i C_{2}(E)
\end{aligned}
$$

Thus by (1)

$$
\Gamma_{2}(E)=\int_{\Omega}|\operatorname{grad} \omega|^{2} d A=2\left|\int \frac{\partial \omega}{\partial z} d z\right|=2 \pi C_{2}(E)
$$

and $\gamma_{2}(E)=\left\{C_{2}(E) / 2 \pi\right\}^{1 / 2}$ by Lemma 1 .

For $p>2$ we prove $\Gamma_{q}(E) \leqq K M_{q}(E)$. Cover $E$ with finitely many disks $\sigma_{i}$ with radius $r_{i}$ so that $\sum r_{i}^{2-q}<M_{q}(E)+\varepsilon$. Let $\sigma_{i}^{\prime}$ be the expanded disks with radius $2 r_{i}$. Let $\omega_{i}(z)$ be continuous, $\omega_{i}(z)=1$ on $\sigma_{i}, \omega_{i}(z)=0$ outside $\sigma_{i}^{\prime}$, and a linear function of the distance to the center of $\sigma_{i}$ for $z$ in $\sigma_{i}^{\prime} \mid \sigma_{i}$. Set $\omega(z)=\operatorname{Max}_{i} \omega_{i}(z)$. Then

$$
\begin{aligned}
\int|\operatorname{grad} \omega|^{q} d A & \leqq \sum_{i} \int_{\sigma i}\left|\operatorname{grad} \omega_{i}\right|^{q} d A \\
& =\sum_{i} 2 \pi \int_{r_{i}}^{2 r_{i}} r_{i}^{-q} r d r=3 \pi \sum r_{i}^{2-q},
\end{aligned}
$$

which proves the inequality.

For the proof in the other direction we assume that $C_{q}(E)>0$. Then there is a unit measure $\mu$ on $E$ such that $\sup _{z} U_{q}^{\mu}(z) \leqq 2 / C_{q}(E)$. The function $\hat{\mu}(z)=\int d \mu(\zeta) /(\zeta-z)$ is analytic off $E, \lim _{z \rightarrow \infty}|z \hat{\mu}(z)|=1$, and $\left\{\int|\hat{\mu}(z)|^{p} d A\right\}^{1 / p} \leqq K / C_{q}(E)^{1 / q}$ by an inequality due to du Plessis [9], and Deny [8]. (A different proof of the inequality is given in [7].) This proves the lemma.

Lemma 3. If $E$ is a continuum with diameter $d$ there are positive constants $K_{1}$ and $K_{2}$, only depending on $p$ (and the distance from $E$ to $\partial \Omega$, for $p=2$ ), such that

$$
\begin{aligned}
K_{1} /(\log 1 / d)^{1 / 2} & \leqq \gamma_{2}(E) \leqq K_{2} /(\log 1 / d)^{1 / 2} ; \\
K_{1} d^{1-2 / p} & \leqq \gamma_{p}(E) \leqq K_{2} d^{1-2 / p}, \quad p>2 .
\end{aligned}
$$

Proof. It is well known that $C_{q}(E)$ is not increased by a contraction of $E$. See e.g. Landkof [19, p. 198]. Thus by Lemma 2 it is enough to compute $C_{q}$ for an interval and $M_{q}$ for a disk, which is easy by means of a homothety.

3. Main results. Theorem 1 is known, and Theorems 2 and 4 are only slight improvements of known theorems, but we include them here in the interest of completeness. See the historical remarks below.

We prefer to give the results in terms of $\gamma_{p}$ rather than the equivalent $\Gamma_{q}$, since $\gamma_{p}$ is what is needed in most of the proofs. 
THEOREM 1. Let $E$ be a bounded measurable set. Then $R^{p}(E)=L_{a}^{p}(E), 1 \leqq p<2$.

THEOREM 2. Let E be compact without interior. Then the following statements are equivalent:

(i) $R^{2}(E)=L^{2}(E)$.

(ii) For almost all $z \in E$ (with respect to plane Lebesgue measure)

$$
\limsup _{\delta \rightarrow 0} C_{2}\left(K_{z}(\delta) \backslash E\right) \delta^{-2}>0 .
$$

(iii) For all open sets $U$ in $\Omega, C_{2}(U \backslash E)=C_{2}(U)$. In particular, $C_{2}\left(K_{z}(\delta) \backslash E\right)$ $=C_{2}\left(K_{z}(\delta)\right) \approx K /(\log 1 / \delta)$ for all $z$ and $\delta>0$.

TheOREM 3. Let $E$ be compact without interior, and let $2<p<\infty$. Then the following statements are equivalent:

(i) $R^{p}(E)=L^{p}(E)$.

(ii) For almost all $z \in E$ (with respect to plane Lebesgue measure)

$$
\limsup _{\delta \rightarrow 0} \gamma_{p}\left(K_{z}(\delta) \backslash E\right) \delta^{-2 / q}=\infty .
$$

(iii) For all bounded open sets $U, \gamma_{p}(U \backslash E)=\gamma_{p}(U)$. In particular, $\gamma_{p}\left(K_{z}(\delta) \backslash E\right)$ $=\gamma_{p}\left(K_{z}(\delta)\right)=K \delta^{1-2 / p}=K \delta^{2 / q-1}$ for all $z$ and $\delta>0$.

THEOREM 4. Let $E$ be compact. Then the following statements are equivalent:

(i) $R^{2}(E)=L_{a}^{2}(E)$.

(ii) For almost all $z \in \partial E \backslash \partial E^{\circ}$ (with respect to plane Lebesgue measure)

$$
\limsup _{\delta \rightarrow 0} C_{2}\left(K_{z}(\delta) \backslash E\right) \delta^{-2}>0,
$$

and for almost all $z \in \partial E^{\circ}$ (with respect to logarithmic capacity)

$$
\int_{0} C_{2}\left(K_{z}(\delta) \backslash E\right) \delta^{-1} d \delta=\infty .
$$

(iii) For all open sets $U$ in $\Omega, C_{2}(U \backslash E)=C_{2}\left(U \backslash E^{\circ}\right)$.

TheOREM 5. Let $E$ be compact, $2<p<\infty$. Then $R^{p}(E)=L_{a}^{p}(E)$ if the following conditions are satisfied:

(i) For almost all $z \in \partial E \backslash \partial E^{\circ}$ (with respect to plane Lebesgue measure)

$$
\limsup _{\delta \rightarrow 0} \gamma_{p}\left(K_{z}(\delta) \backslash E\right) \delta^{-2 / q}=\infty .
$$

(ii) There is a denumerable sequence of nondecreasing functions $\left\{h_{i}\right\}$ such that for almost all $z \in \partial E^{\circ}$ (with respect to $\gamma_{p}$ )

$$
\liminf _{\delta \rightarrow 0} \frac{\gamma_{p}\left(K_{z}(\delta) \backslash E\right)}{h_{i}(\delta)}>0, \text { for some } i,
$$

$h_{i}^{p}(\delta) \delta^{1-p}$ are nonincreasing, and

$$
\int_{0} \operatorname{Min}\left(\frac{1}{\delta \log 1 / \delta}, \frac{h_{i}^{p}(\delta)}{\delta^{p-1}}\right) d \delta=\infty .
$$


Lemma 3 gives the following corollary to Theorems 4 and 5 .

Corollary. Let $E$ be compact, $2 \leqq p<\infty$. Then $R^{p}(E)=L_{a}^{p}(E)$ if the inner boundary $\partial^{\prime} E$ (i.e. the set of points in $\partial E$ which are not on the boundary of any component of $\mathscr{C} E)$ satisfies $\gamma_{p}\left(\partial^{\prime} E\right)=0$.

In fact, we can take $h_{i}(\delta)=\delta^{1-2 / p}$ when $p>2$.

THEOREM 6. Let $E$ be compact, $2<p<\infty$. If $R^{p}(E)=L_{a}^{p}(E)$, then

(i) $\gamma_{p}(U \backslash E)=\gamma_{p}\left(U \backslash E^{\circ}\right)$ for all bounded open sets $U$;

(ii) for almost all $z \in \partial E^{\circ}$ (with respect to $C_{q}$ )

$$
\int_{0} \frac{\gamma_{p}^{q}\left(K_{z}(\delta) \backslash E\right)}{\delta^{3-q}} d \delta=\infty
$$

(iii) for any nondecreasing function $h$ such that

$$
\int_{0} \frac{h^{q}(\delta)}{\delta^{3-q}} d \delta<\infty
$$

the set $F \subset \partial E^{\circ}$ where $\lim _{\delta \rightarrow 0} \gamma_{p}\left(K_{z}(\delta) \backslash E\right) / h(\delta)=0$ satisfies $\gamma_{p}(F)=0$.

REMARK 1. Lemma 2 shows that Theorems 3 and 5 give sufficient conditions for approximation in terms of potential theoretic capacity. It will be seen from the proof that (3) can then be replaced by the somewhat weaker sufficient condition that lim $\sup _{\delta \rightarrow 0} C_{q}\left(K_{z}(\delta) \backslash E\right) \delta^{-2}>0$. If $\gamma_{p}^{q}$ is replaced by $C_{q},(4)$ and (7) are of course the integral form of Wiener's condition for regular points. See e.g. [19, p. 356].

REMARK 2. Theorem 1 is due to Sinanjan [22] in the case of no interior points, to Bers [2] for $p=1$ (and open sets), and to Havin [15] for $1<p<2$. For $p=2$ a somewhat weaker necessary and sufficient condition than the equivalence of (i) and (ii) in Theorems 2 and 4 is due to Havin [15]. A necessary and sufficient condition in the case of no interior points (and $p \geqq 2$ ) had been given earlier by Sinanjan [23], who also gave a sufficient condition for approximation in the general case and $p \geqq 2$. Sinanjan's results are included in ours. Related problems were also studied in Brennan [3]. Bagby [1], [1a] has obtained results related to ours.

The equivalence of (ii) and (iii) in Theorem 2 (and uniform approximation of all continuous functions by harmonic functions) was proved by Gončar [12], [13], [14], and Lysenko and Pisarevskiǐ [20]. This phenomenon, the instability of capacity, was discovered by Vituškin for analytic capacity. See [26, Chapter VI:1].

We will treat all $p, 1 \leqq p<\infty$, simultaneously. The bulk of the paper will be devoted to the proof of Theorem 5 . In $\S 4$ we prove that functions in $L^{p}(\partial E)$ can be approximated by rational functions with poles off $E$, and in $\S 5$ we use this result to prove the general result. In $\S 6$, finally, we prove Theorem 6 .

\section{Approximation on nowhere dense sets.}

THEOREM 7. Let $E$ be compact. Then rational functions with poles off $E$ are dense in $L^{p}(\partial E), 1 \leqq p<2$. 
THEOREM 8. Let E be compact. Then rational functions with poles off $E$ are dense in $L^{p}(\partial E), 2 \leqq p<\infty$, if for almost all $z \in \partial E$ (with respect to Lebesgue measure) $\lim \sup _{\delta \rightarrow 0} \gamma_{p}^{q}\left(K_{z}(\delta) \backslash E\right) \delta^{-2}=\infty(>0$ for $p=2)$.

Theorem 7 is easy. In fact, it is enough to prove that if $g \in L^{q}(\partial E)$ and the Cauchy transform $\hat{g}(z)=\int_{\partial E} g(\zeta) /(\zeta-z) d A=0$ for all $z \notin E$, then $g \equiv 0$. But $\hat{g}$ is continuous, being the convolution of $g \in L^{q}$ and $z^{-1} \in L_{\text {lcc }}^{p}$. It follows that $\hat{g}(z)=0$ on $\partial E$, and since $\hat{g}$ is analytic everywhere off $\partial E$ and $\hat{g}(\infty)=0$, the maximum principle implies that $\hat{g} \equiv 0$. It is well known that this implies $g \equiv 0$. (See e.g. [6, Lemma 5].)

In order to prove Theorem 8 we will estimate $\hat{g}(z)$ when $g \in L^{q}(\partial E)$ and $\hat{g}(z)=0$ off $E$. We need a few lemmas.

LEMMA 4. Let $2<p<\infty$. Let $F$ be a compact set with connected complement and assume $\gamma_{p}(F)>0$. Let $f$ be analytic outside $F, f(\infty)=0$, and $\|f\|_{p}^{p}=\int_{\mathscr{C} F}|f|^{p} d A<\infty$. Suppose $F \subset\{|z| \leqq \delta\}$.

Then for $|z|>r \delta>\delta$

$$
|f(z)| \leqq K \frac{\gamma_{p}(F)}{|z|-r \delta}\|f\|_{p}
$$

where $K$ only depends on $p$ and $r$.

Proof. Cf. Sinanjan [23, Lemma 2.1]. Fix $z,|z|>r \delta>\delta$, and consider $\Phi(\zeta)$ $=(f(\zeta)-f(z)) /(\zeta-z)$. Then $\Phi$ is analytic outside $F, \Phi(\infty)=0$, and $\lim _{\zeta \rightarrow \infty}|\zeta \Phi(\zeta)|$ $=|f(z)|$. Therefore, by definition, $|f(z)| \leqq \gamma_{p}(F)\|\Phi\|_{p}$.

We assume that $\|f\|_{p}=1$ and claim that $\|\Phi\|_{p} \leqq K /(|z|-r \delta)$.

For $|\zeta| \geqq r \delta$ we have

$$
|f(\zeta)|^{p} \leqq 1 / \pi(r-1)^{2} \delta^{2}
$$

by the mean value property. Thus, for $|\zeta|=r \delta$

$$
|\Phi(\zeta)| \leqq \frac{|f(\zeta)|+|f(z)|}{|z|-r \delta} \leqq \frac{2}{\pi^{1 / p}(r-1)^{2 / p} \delta^{2 / p}} \cdot \frac{1}{|z|-r \delta} .
$$

By Schwarz's lemma for $|\zeta| \geqq r \delta$

$$
|\Phi(\zeta)| \leqq K \cdot\left(\delta^{1-2 / p} /|\zeta|\right) \cdot 1 /(|z|-r \delta) .
$$

Thus

$$
\int_{|\zeta| \geqq r \delta}|\Phi(\zeta)|^{p} d A \leqq K \frac{\delta^{p-2}}{(|z|-r \delta)^{p}} \int_{|\zeta| \geqq r \delta} \frac{d A}{|\zeta|^{p}}=\frac{K}{(|z|-r \delta)^{p}} .
$$

On the other hand

$$
\begin{aligned}
\int_{|\zeta| \leqq r \delta}|\Phi(\zeta)|^{p} d A & \leqq \frac{K}{(|z|-r \delta)^{p}}\left(\|f\|_{p}^{p}+|f(z)|^{p}(r \delta)^{2}\right) \\
& \leqq \frac{K}{(|z|-r \delta)^{p}}\left(1+\left(\frac{r}{r-1}\right)^{2}\right)
\end{aligned}
$$

which proves the lemma. 
The above lemma implies the following variant of a lemma of Mergeljan and Vituškin. A weaker form of it, using a smaller analytic $p$-capacity, was given by Sinanjan [23, Lemma 2.1].

Lemma 5. Let $2 \leqq p<\infty$, and let $F \subset\{|z| \leqq \delta\}$ be compact with connected complement. If $C_{q}(F)>0$ or $\gamma_{p}(F)>0$ there exists a function $h$, analytic outside $F$ with $h(\infty)=0$, and constants $K$ depending only on $p$, such that $\|h\|_{p} \leqq K / C_{q}(F)^{1 / q}$ or $\|h\|_{p} \leqq K / \gamma_{p}(F)$, respectively, and $\left|\zeta^{-1}-h(\zeta)\right| \leqq K \delta /|\zeta|^{2}$ for all $\zeta,|\zeta| \geqq 2 \delta$.

Proof. First assume $C_{q}(F)>0,1<q \leqq 2$, and let $\mu$ be the corresponding unit equilibrium measure for $F$. Choose $h(\zeta)=\int d \mu(s) /(s-\zeta)$. By the inequalities of $\mathrm{du}$ Plessis [9] and Deny [8] referred to earlier, $\|h\|_{p} \leqq K / C_{q}(F)^{1 / q}$. Moreover, $\lim _{\zeta \rightarrow \infty} \zeta h(\zeta)=1$, so $\lim _{\zeta \rightarrow \infty} \zeta^{2}\left(\zeta^{-1}-h(\zeta)\right)$ exists. For $|\zeta|=2 \delta$ clearly $\left|\zeta^{2}\left(\zeta^{-1}-h(\zeta)\right)\right|$ $\leqq 6 \delta$. The inequality for $|\zeta| \geqq 2 \delta$ follows from the maximum principle.

Now assume $\gamma_{p}(F)>0, p>2$, and let $h$ be analytic outside $F, h(\infty)=0$, $\lim _{\zeta \rightarrow \infty} \zeta h(\zeta)=1$, and $\|h\|_{p} \leqq 2 / \gamma_{p}(F)$. The lemma follows as above if Lemma 4 is applied to $h$ for $|\zeta|=2 \delta$.

The following lemma will be crucial also in the following section.

Lemma 6. Let $E$ be compact, $2 \leqq p<\infty$. Let $z \in E$ and suppose $F \subset K_{z}(\delta) \backslash E$ is compact, $\gamma_{p}(F)>0$. Let $g \in L^{q}(E)$, and suppose $\hat{g}(\zeta)=0$ for all $\zeta \notin E$. Then

$$
|\hat{g}(z)| \leqq K\left(g^{*}(z) \delta \log \frac{1}{\delta}+\left\{\int_{|\zeta-z|<2 \delta}|g(\zeta)|^{q} d A\right\}^{1 / q} \frac{1}{\gamma_{p}(F)}\right)
$$

where

$$
g^{*}(z)=\sup _{r} \frac{1}{\pi r^{2}} \int_{|\zeta-z|<r ; \zeta \in E}|g(\zeta)| d A,
$$

and $K$ depends only on $q$ and the diameter of $E$.

$$
\begin{aligned}
& \text { If } \left.C_{q}(F)>0 \text { and } 1<q \leqq 2 \text {, then (if } g(z)=\lim _{r \rightarrow 0}\left(1 / \pi r^{2}\right) \int_{|\zeta-z|<r ; \zeta \in E} g(\zeta) d A\right) \\
& \qquad|\hat{g}(z)| \leqq K\left(g^{*}(z) \delta \log \frac{1}{\delta}+\left\{\int_{|\zeta-z|<2 \delta}|g(\zeta)-g(z)|^{q} d A\right\}^{1 / q} \frac{1}{C_{q}(F)^{1 / q}}\right) .
\end{aligned}
$$

Proof. Cf. the proof of [6, Lemma 1]. Assume $\gamma_{p}(F)>0$, put $z=0$, and let $h$ be the function given in Lemma 5. We extend the definition of $g$ by setting $g(\zeta)=0$, $\zeta \notin E$. By Runge's theorem $\int g h d A=0$, so $\hat{g}(0)=\int g(\zeta)(1 / \zeta-h(\zeta)) d A$. By Lemma 5

Here

$$
\begin{aligned}
|\hat{g}(0)| & \leqq \int_{|\zeta| \leqq 2 \delta} \frac{|g(\zeta)|}{|\zeta|} d A+\int_{|\zeta| \leqq 2 \delta}|g(\zeta) h(\zeta)| d A+K \delta \int_{|\zeta|>2 \delta} \frac{|g(\zeta)|}{|\zeta|^{2}} d A \\
& =I_{1}+I_{2}+I_{3} .
\end{aligned}
$$

$$
\begin{aligned}
I_{1} & =\sum_{n=0}^{\infty} \int_{\delta \cdot 2^{-n} \leqq|\zeta|<\delta \cdot 2^{-n+1}} \frac{|g(\zeta)|}{|\zeta|} d A \\
& \leqq \sum_{n=0}^{\infty} \delta \cdot 2^{n} \cdot \int_{|\zeta|<\delta \cdot 2^{-n+1}}|g(\zeta)| d A \leqq K \delta g^{*}(0)
\end{aligned}
$$


Similarly

$$
I_{3}=\sum_{n=1}^{N} \int_{\delta \cdot 2^{n} \leqq|\zeta| \leqq \delta \cdot 2^{n+1}} \frac{|g(\zeta)|}{|\zeta|^{2}} d A \leqq A g^{*}(0) \delta N
$$

where $N$ is chosen so that $R<\delta 2^{N+1} \leqq 2 R$ if $E$ is contained in $\{|\zeta| \leqq R\}$.

An application of Hölder's inequality to $I_{2}$ completes the proof of the first inequality.

Now assume $C_{q}(F)>0$ and choose $h(\zeta)=\int d \mu(s) /(s-\zeta)$ as in Lemma 5. We now write

$$
\begin{aligned}
I_{2} & =\left|\int_{|\zeta| \leqq 2 \delta} g(\zeta) h(\zeta) d A\right| \\
& \leqq \int_{|\zeta| \leqq 2 \delta}|g(\zeta)-g(0)||h(\zeta)| d A+|g(0)| \int_{|\zeta| \leqq 2 \delta}|h(\zeta)| d A \\
& \leqq\left\{\int_{|\zeta| \leqq 2 \delta}|g(\zeta)-g(0)|^{q} d A\right\}^{1 / q} \frac{K}{C_{q}(F)^{1 / q}}+|g(0)| \int d \mu(s) \int_{|\zeta| \leqq 2 \delta} \frac{d A_{\zeta}}{|s-\zeta|}
\end{aligned}
$$

The last integral is less than $K \delta$, and this proves the lemma.

We now assume that $g \in L^{q}(\partial E), 1<q \leqq 2$, and $\hat{g}(\zeta)=0, \zeta \notin E$. We shall prove that $g=0$ almost everywhere if $E$ has the property in Theorem 8 . We observe that Lemma 6 implies that $\hat{g}(\zeta)=0$ almost everywhere on $\partial E$, and this proves the asssertion when $E$ has no interior.

REMARK. The same argument as in Lemma 6 applies to measures and gives a short proof of the following theorem of Vituškin (see [26, Chapter VI:2], where a slightly stronger result is proved).

$R(E)=C(E)$ if for almost all $z \in E$ (with respect to plane Lebesgue measure) $\lim \sup _{\delta \rightarrow 0} \gamma\left(K_{z}(\delta) \backslash E\right) / \delta^{2}=\infty$.

Here $\gamma$ is analytic capacity, and $R(E)$ is the uniform closure of the rational functions.

To prove Theorem 8 in the general case we need the following two lemmas.

Lemma 7. Let $g \in L^{q}(\partial E), 1<q \leqq 2$, let $z$ be given, and suppose $\hat{g}(\zeta)=0$ in some set $F \subset K_{z}(\delta)$ with mes $F>\frac{7}{8} \pi \delta^{2}$. Then $|\hat{g}(z)| \leqq K g^{*}(z) \delta$.

Proof. Suppose $z=0$. The intersection of $F$ and a sector

$$
S_{\alpha}=\left\{r e^{i \theta} ; 0 \leqq r \leqq \delta, \alpha \leqq 0 \leqq \alpha+\pi / 2\right\}
$$

has measure at least $\frac{1}{8} \pi \delta^{2}$. Let $\chi_{1}$ and $\chi_{2}$ be characteristic functions of subsets of $S_{\pi / 4}$ and $S_{5 \pi / 4}$ with measure $\frac{1}{8} \pi \delta^{2}$. It is then easily seen that we can determine $a(\zeta)=a_{1} \chi_{1}(\zeta)+a_{2} \chi_{2}(\zeta)$ so that $\int a(\zeta) d A=1, \int \zeta a(\zeta) d A=0$, and then $|a(\zeta)| \leqq K \delta^{-2}$. 
Now put $h(\zeta)=\int a(s) d A_{s} /(\zeta-s)$. Then sup $|h(\zeta)| \leqq K \delta^{-1}$, and as in Lemma 5 we find $|1 / \zeta-h(\zeta)| \leqq K \delta^{2} /|\zeta|^{3}$, for $|\zeta| \geqq 2 \delta$. Now $\int g(\zeta) h(\zeta) d A=-\int a(s) \hat{g}(s) d A=0$, so

$$
\begin{aligned}
|\hat{g}(0)| & =\left|\int g(\zeta)\left(\frac{1}{\zeta}-h(\zeta)\right) d A\right| \\
& \leqq K \int_{|\zeta| \leqq 2 \delta} \frac{|g(\zeta)|}{|\zeta|} d A+K \delta^{2} \int_{|\zeta| \geqq 2 \delta} \frac{|g(\zeta)|}{|\zeta|^{3}} d A \leqq K g^{*}(0) \delta
\end{aligned}
$$

by estimates similar to those in the proof of Lemma 6 .

The following is a stronger version of a well-known lemma (see e.g. [6, Lemma 5]).

LEMMA 8. If $g \in L^{q}(\partial E), 1<q \leqq 2$, and $\hat{g}(\zeta)=0$ almost everywhere on $\partial E$, then $g(\zeta)=0$ almost everywhere.

Proof. As usual we put $g(\zeta)=0, \zeta \notin \partial E$. By Fubini's and Cauchy's theorems

$$
\frac{1}{2 \pi i} \int_{|\zeta-z|=\delta} \hat{g}(\zeta) d \zeta=\frac{1}{2 \pi i} \int g(s) d A \int_{|\zeta-z|=\delta} \frac{d \zeta}{s-\zeta}=-\int_{|s-z| \leqq \delta} g(s) d A
$$

for almost all $z$ and $\delta$. Therefore the lemma will follow if we show that for almost all $z \in \partial E$

$$
\liminf _{\delta \rightarrow 0} \frac{1}{\delta^{2}} \int_{|\zeta-z|=\delta}|\hat{g}(\zeta)||d \zeta|=0 .
$$

Let $z$ be a density point for $\partial E$, and assume moreover that

$$
\lim _{\delta \rightarrow 0} \frac{1}{\pi \delta^{2}} \int_{|\zeta-z| \leqq \delta ; \zeta \notin \partial E} g^{*}(\zeta) d A=0 .
$$

This is true for almost all $z \in \partial E$, since $g^{*} \in L^{q}(\partial E)$. (See e.g. [24, Theorem I.1], for a simple proof.)

By Lemma 7 we get for sufficiently small $\delta$

$$
\int_{|\zeta-z| \leqq \delta}|\hat{g}(\zeta)| d A \leqq K \int_{|\zeta-z| \leqq \delta \zeta \zeta \notin \partial E} g^{*}(\zeta)|\zeta-z| d A=o\left(\delta^{3}\right)
$$

by the choice of $z$. It follows that

$$
\liminf _{\delta \rightarrow 0} \frac{1}{\delta^{2}} \int_{|\zeta-z|=\delta}|\hat{g}(\zeta)||d \zeta|=0
$$

which proves the lemma and Theorem 8.

5. Approximation on arbitrary compact sets. We shall now apply the results of the preceding section to the general case. Suppose $g \in L^{q}(E)$ and $\hat{g}(z)=0$ for $z \in \mathscr{C} E$. We will show that $\int_{E} f g d A=0$ for $f \in L_{a}^{p}(E)$.

We first observe that it suffices to prove that $\int_{E^{\circ}} f g d A=0$ for $f \in L_{a}^{p}(E)$. In fact, this implies that

$$
\int_{E^{\circ}} \frac{g(\zeta) d A}{\zeta-z}=0, \quad z \notin E
$$


and therefore

$$
\int_{\partial E} \frac{g(\zeta) d A}{\zeta-z}=0, \quad z \notin E
$$

But then $g(\zeta)=0$ a.e. on $\partial E$ by Theorems 7 and 8 , so $\int_{E} f g d A=\int_{E^{\circ}} f g d A=0$.

For $p=2$, (4) implies that $\int_{E^{\circ}} f g d A=0$ as in [15], which proves that (ii) implies (i) in Theorem 4. Now let $p \neq 2$. Following Bers [2], we let $\left\{\omega_{n}\right\}$ be an increasing sequence of functions with compact support in $E^{\circ}$ such that $0 \leqq \omega_{n} \leqq 1$ and $\lim _{n \rightarrow \infty} \omega_{n}$ $=1$ for almost all $z \in E^{\circ}$. It is sufficient to prove that $\lim _{n \rightarrow \infty} \int \omega_{n} f g d A=0$ for a suitable choice of the sequence. But by Green's theorem and the analyticity of $f$

and

$$
\int \omega_{n} f g d A=-\frac{1}{\pi} \int \omega_{n} f \frac{\partial \hat{g}}{\partial \bar{z}} d A=\frac{1}{\pi} \int f \frac{\partial \omega_{n}}{\partial \bar{z}} \hat{g} d A
$$

$$
\left|\int f \frac{\partial \omega_{n}}{\partial \bar{z}} \hat{g} d A\right| \leqq\|f\|_{p}\left\|\hat{g} \operatorname{grad} \omega_{n}\right\|_{q} .
$$

We shall show that the $\omega_{n}$ can be chosen so that $\| \hat{g}$ grad $\omega_{n} \|_{q} \rightarrow 0, n \rightarrow \infty$.

The proof will follow from the following lemmas. Lemma 9 is a counterpart to Lemma 6 for $q>2$.

Lemma 9. If $g \in L^{q}(E), 2<q<\infty$, then for $\left|z_{1}-z\right| \leqq \delta$

$$
\left|\hat{g}(z)-\hat{g}\left(z_{1}\right)\right| \leqq K\left(g^{*}(z) \delta \log \frac{1}{\delta}+\left\{\int_{|\zeta-z|<2 \delta}|g(\zeta)|^{q} d A\right\}^{1 / q} \cdot \delta^{1-2 / q}\right) .
$$

For $q=\infty$

$$
\left|\hat{g}(z)-\hat{g}\left(z_{1}\right)\right| \leqq K\|g\|_{\infty} \delta \log \frac{1}{\delta} .
$$

The proof is similar to that of Lemma 6 and is omitted.

LEMMA 10. Let $F$ be a compact subset of $\partial E^{\circ}$ such that the assumptions in Theorem 5 are satisfied for all $z \in F$. $\left(F=\partial E^{\circ}\right.$ for $p<2$.) Then for given $\varepsilon>0$ there exists a function $\omega, 0 \leqq \omega(z) \leqq 1$, such that $\omega(z)=0$ in a neighborhood of $F$, and $\omega(z)=1$ outside an $\varepsilon$-neighborhood of $F$, and $\|\hat{g} \operatorname{grad} \omega\|_{q}<\varepsilon$.

Proof. By considering $h_{i} / n, n=1,2, \ldots$, and reenumerating, we can assume that, for each $z \in F, \gamma_{p}\left(K_{z}(\delta) \backslash E\right) \geqq h_{m}(\delta)$ for all $\delta \leqq 1 / m$, for some $m$. Let $F_{m}$ be the set of such $z$, so $F=\bigcup_{1}^{\infty} F_{m}$.

We now fix $m$, and we shall construct a function $\omega_{m}$ which is 0 in a neighborhood of $\bar{F}_{m}, 1$ outside a neighborhood of $\bar{F}_{m}$ and is such that $\left\|\hat{g} \operatorname{grad} \omega_{m}\right\|_{q}<\varepsilon / 2^{m}$.

By Lemma 6 applied to a point $z$ at distance $\delta(z)$ from $F_{m}$ and to the disk $K_{z}(2 \delta(z))$, we obtain

$$
|\hat{g}(z)| \leqq K\left(g^{*}(z) \delta(z) \log \frac{1}{\delta(z)}+\left\{\int_{|\zeta-z|<4 \delta(z)}|g(\zeta)|^{q} d A\right\}^{1 / q} \frac{1}{h_{m}(\delta(z))}\right) .
$$

(Put $h(z)=\delta^{2 / q-1}$ for $p<2$ by Lemma 9.) 
To construct $\omega_{m}$ we now apply an idea of Ahlfors which appears in [2].

Let $\lambda(t), t>0$, be a nonincreasing function such that $\int_{r} \lambda(t) d t=\Lambda(r) \rightarrow \infty$ as $r \rightarrow+0$. Let $k(t), t>0$, be a continuous piecewise linear function such that $k(t)=0$ for $t \leqq 1, k(t)=1$ for $t \geqq 2$, and $\left|k^{\prime}(t)\right| \leqq 1$. Put $K_{n}(t)=k(n / \Lambda(t)), n=1,2, \ldots$ Then $K_{n}^{\prime}(t)=k^{\prime}(n / \Lambda(t)) \cdot\left(-n / \Lambda^{2}(t)\right) \cdot(-\lambda(t))$, so $K_{n}^{\prime}(t)=0$ except for $0<t_{n}^{\prime} \leqq t \leqq t_{n}^{\prime \prime}$. For $t_{n}^{\prime} \leqq t \leqq t_{n}^{\prime \prime}, \lambda(t) / n \leqq K_{n}^{\prime}(t) \leqq 4 \lambda(t) / n$, and $\int_{t^{\prime}}^{t^{\prime \prime}} K_{n}^{\prime}(t) d t=1$.

We now choose $\lambda(t)=\operatorname{Min}\left(1 /(t \log 1 / t), h_{m}^{p}(t) / t^{p-1}\right)$, and put $\omega_{m}(z)=K_{n}(\delta(z))$, where $n$ is to be determined. Then $\left|\operatorname{grad} \omega_{m}(z)\right| \leqq K_{n}^{\prime}(\delta(z))$. A short calculation gives

$$
\left|\hat{g}(z) \operatorname{grad} \omega_{m}(z)\right|^{q} \leqq \frac{K}{n^{q-1}}\left(g^{*}(z)^{q}+\frac{K_{n}^{\prime}(\delta(z))}{\delta(z)} \int_{|\zeta-z|<4 \delta(z)}|g(\zeta)|^{q} d A\right) .
$$

By the Hardy maximal theorem (see e.g. [24, Theorem I.1] for a simple proof) we only have to estimate the integral of the second term. We find, since $\lambda$ is nonincreasing,

$$
\begin{aligned}
& \int \frac{K_{n}^{\prime}(\delta(z))}{\delta(z)} d A_{z} \int_{|\zeta-z|<4 \delta(z)}|g(\zeta)|^{q} d A_{\zeta} \\
& \leqq \frac{K}{n} \int d A_{z} \int_{|w|<4 \delta(z)} \operatorname{Min}\left(\frac{\lambda\left(t_{n}^{\prime}\right)}{t_{n}^{\prime}}, \frac{\lambda(|w| / 4)}{|w| / 4}\right)|g(z+w)|^{q} d A_{w} \\
& \leqq \frac{K}{n} \int \operatorname{Min}\left(\frac{\lambda\left(t_{n}^{\prime}\right)}{t_{n}^{\prime}}, \frac{\lambda(|w| / 4)}{|w| / 4}\right) d A_{w} \int|g(z)|^{q} d A_{z} \\
& \leqq \frac{K}{n}\left(\lambda\left(t_{n}^{\prime}\right) \cdot t_{n}^{\prime}+\int \lambda(t) d t\right)\|g\|_{q}^{q} \leqq K\|g\|_{q}^{q}
\end{aligned}
$$

since $\lambda(t) \leqq 1 / t$. Thus $\left\|\hat{g} \operatorname{grad} \omega_{m}\right\|_{q}<\varepsilon / 2^{m}$ if $n$ is large enough.

Now put $\omega^{(m)}=\prod_{1}^{m} \omega_{i}$. Then $\left|\operatorname{grad} \omega^{(m)}\right| \leqq \sum_{1}^{m}\left|\operatorname{grad} \omega_{i}\right|$, so $\left\|\hat{g} \operatorname{grad} \omega^{(m)}\right\|_{q}<\varepsilon$. Also $\omega^{(m)}=0$ in a neighborhood $O_{m}$ of $\bar{F}_{m}$, and the sequence $\left\{O_{m}\right\}$ is increasing. Therefore, by the compactness of $F, F \subset O_{N}$ for some $N$. We choose $\omega=\omega^{(N)}$, and this finishes the proof of the lemma and of Theorem 1.

LEMMA 11. Let $2 \leqq p<\infty$ and let $F$ be a Borel subset of $\partial E^{\circ}$ with $\gamma_{p}(F)=0$. Then there exist functions $\omega_{n}, 0 \leqq \omega_{n}(z) \leqq 1$, such that $\lim _{n \rightarrow \infty}\left\|\hat{g} \operatorname{grad} \omega_{n}\right\|_{q}=0$, $\lim _{n \rightarrow \infty} \omega_{n}(z)=1$ in $E^{\circ}$, and $\omega_{n}(z)=0$ in a neighborhood of $F$.

Proof. By Lemma 1, and since Borel sets are capacitable for $\Gamma_{q}$ (see [29]), there is for every $n$ an open neighborhood $O_{n}$ of $F$ such that $\Gamma_{q}\left(O_{n}\right)^{1 / q} \leqq \varepsilon_{n} / n$, and $\lim _{n \rightarrow \infty} \varepsilon_{n}=0$. Then there is an increasing sequence of compact sets, $K_{i} \subset O_{n}$, $O_{n}=\bigcup_{1}^{\infty} K_{i}$, and functions $\varphi_{n}^{(i)}, \varphi_{n}^{(i)}(z)=1$ on $K_{i}$, such that $\left\|\operatorname{grad} \varphi_{n}^{(i)}\right\|_{q} \leqq \varepsilon_{n} / n$. We choose a weakly convergent subsequence of $\left\{\varphi_{n}^{(i)}\right\}_{i}$, and denote the limit function by $\varphi_{n}$. Then $\varphi_{n}(z)=1$ on $O_{n}$, and $\left\|\operatorname{grad} \varphi_{n}\right\|_{q} \leqq \varepsilon_{n} / n$. Moreover (see [29]), $\Gamma_{q}^{1 / q}\left\{z ; \varphi_{n}(z)>\frac{1}{2}\right\}$ $\leqq K \varepsilon_{n} / n$. Set $\varphi_{n}^{\prime}(z)=2 \operatorname{Max}\left(\varphi_{n}(z)-\frac{1}{2}, 0\right)$. It follows that $\lim _{n \rightarrow \infty} \varphi_{n}^{\prime}(z)=0$ a.e. in $E^{\circ}$, at least for a subsequence, which we still denote by $\left\{\varphi_{n}^{\prime}\right\}$. 
Now set $S_{n}=\{z ;|\hat{g}(z)|>n\}$. By the Calderón-Zygmund theory for singular integrals (see [3]) $\|\operatorname{grad} \hat{g}\|_{q} \leqq K\|g\|_{q}$, and moreover $|\operatorname{grad}| \hat{g}|| \leqq|\operatorname{grad} \hat{g}|$ almost everywhere, so $\Gamma_{q}\left(S_{n}\right)^{1 / q} \leqq(K / n)\|g\|_{q}$.

Define $\psi_{n}(z)=0$ on $S_{n}, \psi_{n}(z)=1$ outside $S_{n / 2}$, and $\psi_{n}(z)=2(1-\hat{g}(z) / n)$ otherwise. It follows that $\left\|\operatorname{grad} \psi_{n}\right\|_{q}=o(1 / n)$, and that $\lim _{n \rightarrow \infty} \psi_{n}(z)=1$ almost everywhere.

We now set $\omega_{n}=\left(1-\varphi_{n}^{\prime}\right)^{2} \psi_{n}^{2}$. Then

$$
\begin{aligned}
\left|\operatorname{grad} \omega_{n}\right| & \leqq 2\left(1-\varphi_{n}\right) \psi_{n}^{2}\left|\operatorname{grad} \varphi_{n}\right|+2\left(1-\varphi_{n}\right)^{2} \psi_{n}\left|\operatorname{grad} \psi_{n}\right| \\
& \leqq 2 \psi_{n}\left(\left|\operatorname{grad} \varphi_{n}\right|+\left|\operatorname{grad} \psi_{n}\right|\right) .
\end{aligned}
$$

We find $\int\left|\hat{g} \psi_{n} \operatorname{grad} \psi_{n}\right|^{q} d A \leqq n^{q}\left\|\operatorname{grad} \psi_{n}\right\|_{q}^{q}<\varepsilon$ for $n$ large enough, and similarly $\int\left|\hat{g} \psi_{n} \operatorname{grad} \varphi_{n}\right|^{q} d A \leqq n^{q}\left\|\operatorname{grad} \varphi_{n}\right\|_{q}^{q}<\varepsilon$. This proves the lemma.

To complete the proof of Theorem 5 we now only have to observe that, by assumption, (5) is satisfied except on a set $G$ with $\gamma_{p}(G)=0$. We choose a function $\omega^{\prime}$ as in Lemma 11 , with $\omega^{\prime}=0$ in a neighborhood $O$ of $G$. Then $\partial E \backslash O$ satisfies the assumptions of Lemma 10, and we choose $\omega^{\prime \prime}=0$ in a neighborhood of $\partial E \backslash O$ as in Lemma 10. We then set $\omega=\omega^{\prime} \omega^{\prime \prime}$, and the result follows.

6. Necessary conditions for approximation. We shall first show that if $R^{p}(E)=L_{a}^{p}(E)$ then $\gamma_{p}(U \backslash E)=\gamma_{p}\left(U \backslash E^{\circ}\right)$ for all open bounded sets $U$. The proof is similar to the corresponding proof for uniform approximation as given e.g. in [28, p. 104].

Lemma 12. Let $U$ be a bounded open set. Suppose that $f \in R^{p}(E), f$ is analytic outside a compact subset of $U$ and that $f(\infty)=0$. Then $f \in R^{p}(\mathscr{C} U \cup E)$.

Proof. By Runge's theorem $(z-a) f$ can be uniformly approximated on $\mathscr{C} U$ for all $a$ by rational functions with poles in $U$. If $a$ is chosen in $U$ it follows that $f \in R^{p}(\mathscr{C} U), p \geqq 2$, where the norm is taken over a fixed disk for $p=2$. The lemma now follows exactly as in [28, 11.7 and 15.9].

Now assume $R^{p}(E)=L^{p}(E)$ and let $U$ be any open set with $\gamma_{p}\left(U \backslash E^{\circ}\right)>0$. Then for given $\varepsilon>0$ there exists a function $f$ which is analytic outside a compact subset of $U \backslash E^{\circ}, \int_{\mathscr{C}\left(U \backslash E^{\circ}\right)}|f|^{p} d A=1$, and $\left|f^{\prime}(\infty)\right| \geqq \gamma_{p}\left(U \backslash E^{\circ}\right)-\varepsilon$. We put $f(z)=0$ for $z \in U \backslash E^{\circ}$.

The restriction of $f$ to $E$ is in $R^{p}(E)$ by assumption, so by Lemma 12 we can find a sequence of rational functions $f_{n}$ with poles in $U \backslash E$ tending to $f$ in $L^{p}(\mathscr{C} U \cup E)$. Clearly $f_{n}^{\prime}(\infty) \rightarrow f^{\prime}(\infty)$, and

$$
\int_{\mathscr{C} \cup \cup E}\left|f_{n}\right|^{p} d A \rightarrow \int_{\mathscr{C} \cup \cup E}|f|^{p} d A=\int_{\mathscr{C} \cup \cup E^{\circ}}|f|^{p} d A=1 .
$$

It follows that $\gamma_{p}(U \backslash E) \geqq \gamma_{p}\left(U \backslash E^{\circ}\right)-\varepsilon$, which proves Theorems 2(iii), 3(iii), 4(iii), and 6(i).

We shall now prove that these statements imply Theorems 4(ii), and 6(ii) and (iii). (Cf. Vituškin [26, Chapter V:2].)

Suppose $\gamma_{p}(U \backslash E)=\gamma_{p}\left(U \backslash E^{\circ}\right)$ for all bounded open $U$, and assume that there is a 
compact set $F \subset \partial E^{\circ}$ with $\gamma_{p}(F)>0$ such that $\int_{0} \gamma_{p}^{q}\left(K_{z}(\delta) \backslash E\right) \delta^{q-3} d \delta<\infty$ for all $z \in F$. Then, for all $z \in F$

$$
\begin{aligned}
\int_{0} \gamma_{p}^{q}\left(K_{z}(\delta) \cap F\right) \delta^{q-3} d \delta & \leqq \int_{0} \gamma_{p}^{q}\left(K_{z}(\delta) \backslash E^{\circ}\right) \delta^{q-3} d \delta \\
& =\int_{0} \gamma_{p}^{q}\left(K_{z}(\delta) \backslash E\right) \delta^{q-3} d \delta<\infty
\end{aligned}
$$

By Lemma 2, $\int_{0} C_{q}\left(K_{z}(\delta) \cap F\right) \delta^{q-3} d \delta<\infty$, and that this implies $C_{q}(F)=0$ is Kellogg's lemma. See e.g. [25, Theorem III:33] or [19, pp. 327 and 353], compare also [26, Chapter III :6].

In order to conclude that the assumptions in Theorem 6(iii) imply that $\gamma_{p}(F)=0$ it is clearly enough to prove the following lemma.

LemMA 13. Let $F$ be compact, $1<q \leqq 2$. If for all $z \in F$ and $\delta, 0<\delta<\delta_{0}$, $\Gamma_{q}\left(K_{z}(\delta) \cap F\right) \leqq h(\delta)$, where $h>0$ is increasing and $\int_{0} h(\delta) \delta^{q-3} d \delta<\infty$, then $\Gamma_{q}(F)=0$.

Proof. Suppose $\Gamma_{q}(F)>0$. For any $d>0$ we can then find a subset $F^{\prime}$ of $F$ with diameter $\leqq d$ and $\Gamma_{q}\left(F^{\prime}\right)>0$. If $F^{\prime}$ is covered by disks $K_{z_{i}}\left(\delta_{i}\right)$ it follows from the assumption and the subadditivity of $\Gamma_{q}$ that $\Gamma_{q}\left(F^{\prime}\right) \leqq \sum_{i} h\left(\delta_{i}\right)$, so $\Gamma_{q}\left(F^{\prime}\right) \leqq M_{h}\left(F^{\prime}\right)$. But on the other hand, it is easily seen from [7, Theorem IV:1] and Lemma 2 above that

$$
M_{h}\left(F^{\prime}\right) \leqq C_{q}\left(F^{\prime}\right) \int_{0}^{d} h(\delta) \delta^{q-3} d \delta \leqq K \Gamma_{q}\left(F^{\prime}\right) \int_{0}^{d} h(\delta) \delta^{q-3} d \delta
$$

which is a contradiction if $d$ is small enough. This proves the lemma.

Theorem 6 follows easily, and this finishes the proof.

\section{REFERENCES}

1. T. Bagby, $L_{p}$ approximation by analytic functions, J. Approximation Theory (to appear). 1a. - Quasi topologies and rational approximation, J. Functional Analysis (submitted).

2. L. Bers, An approximation theorem, J. Analyse Math. 14 (1965), 1-4. MR 31 \#2545.

3. J. Brennan, Invariant subspaces and rational approximation, J. Functional Analysis 7 (1971), 285-310.

4. A. P. Calderón and A. Zygmund, On the existence of certain singular integrals, Acta Math. 88 (1952), 85-139. MR 14, 637.

5. T. Carleman, Uber die Approximation analytischer Funktionen durch lineare Aggregate von vorgegebenen Potenzen, Ark. Mat. Astr. Fys. 17 (1923), 1-30.

6. L. Carleson, Mergelyan's theorem on uniform polynomial approximation, Math. Scand. 15 (1964), 167-175. MR 33 \#6368.

7. - Selected problems on exceptional sets, Van Nostrand Math. Studies, no. 13, Van Nostrand, Princeton, N. J., 1967. MR 37 \#1576.

8. J. Deny, Sur la convergence de certaines intégrales de la théorie du potentiel, Arch. Math. 5 (1954), 367-370. MR 16, 589.

9. N. du Plessis, A theorem about fractional integrals, Proc. Amer. Math. Soc. 3 (1952), 892-898. MR 14, 546. 
10. B. Fuglede, On generalized potentials of functions in the Lebesgue classes, Math. Scand. 8 (1960), 287-304. MR 28 \#2241.

11. T. W. Gamelin, Uniform algebras, Prentice-Hall, Englewood Cliffs, N. J., 1969.

12. A. A. Gončar, On the uniform approximation of continuous functions by harmonic functions, Izv. Akad. Nauk SSSR Ser. Mat. 27 (1963), 1239-1250. (Russian) MR 28 \#2231.

13. - On the approximation of continuous functions by harmonic functions, Dokl. Akad. Nauk SSSR 154 (1964), 503-506= Soviet Math. Dokl. 5 (1964), 105-109. MR 28 \#2390.

14. - On the property of instability of harmonic capacity, Dokl. Akad. Nauk SSSR 165 (1965), 479-481 = Soviet Math. Dokl. 6 (1965), 1458-1460. MR 32 \#7779.

15. V. P. Havin, Approximation in the mean by analytic functions, Dokl. Akad. Nauk SSSR 178 (1968), 1025-1028 = Soviet Math. Dokl. 9 (1968), 245-248. MR 37 \#429.

16. V. P. Havin and V. G. Maz'ja, On approximation in the mean by analytic functions, Vestnik Leningrad. Univ. 23 (1968), no. 13, 62-74. (Russian) MR 38 \#3443.

17. S. Ja. Havinson, Extremal problems for certain classes of analytic functions in finitely connected regions, Mat. Sb. 36 (78) (1955), 445-478; English transl., Amer. Math. Soc. Transl. (2) 5 (1957), 1-33. MR 17, 247; MR 18, 728.

18. L. I. Hedberg, Weighted mean approximation in Carathéodory regions, Math. Scand. 23 (1968), 113-122. MR 41 \#2028.

19. N. S. Landkof, Principles of modern potential theory, "Nauka", Moscow, 1966. (Russian) MR 35 \#5644.

20. Ju. A. Lysenko and B. M. Pisarevskir, The instability of harmonic capacity and the approximation of continuous functions by harmonic functions, Mat. Sb. 76 (118) (1968), 52-71 = Math. USSR Sb. 5 (1968), 53-72. MR 38 \#2497.

21. S. N. Mergeljan, On the completeness of systems of analytic functions, Uspehi Mat. Nauk 8 (1953), no. 4 (56), 3-63; English transl., Amer. Math. Soc. Transl. (2) 19 (1962), 109-166. MR 15, 411; MR 24 \#A1410.

22. S. O. Sinanjan, The uniqueness property of analytic functions on closed sets without interior points, Sibirsk. Mat. Ž. 6 (1965), 1365-1381. (Russian) MR 33 \#290.

23. - Approximation by polynomials and analytic functions in the areal mean, Mat. Sb. 69 (111) (1966), 546-578; English transl., Amer. Math. Soc. Transl. (2) 74 (1968), 91-124. MR 35 \#389.

24. E. M. Stein, Singular integrals and differentiability properties of functions, Princeton Univ. Press, Princeton, N. J., 1970.

25. M. Tsuji, Potential theory in modern function theory, Maruzen, Tokyo, 1959. MR 22 \#5712.

26. A. G. Vituškin, Analytic capacity of sets in problems of approximation theory, Uspehi Mat. Nauk 22 (1967), 141-199= Russian Math. Surveys 22 (1967), 139-200. MR 37 \#5404.

27. H. Wallin, A connection between $\alpha$-capacity and $L^{p}$-classes of differentiable functions, Ark. Mat. 5 (1963/65), 331-341. MR 36 \#417.

28. L. Zalcman, Analytic capacity and rational approximation, Lecture Notes in Math., no. 50, Springer-Verlag, Berlin and New York, 1968. MR 37 \#3018.

29. W. P. Ziemer, Extremal length as a capacity, Michigan Math. J. 17 (1970), 117-128.

Department of Mathematics, Uppsala University, Uppsala, Sweden 